\title{
Ajustamento da criança à separação ou divórcio dos pais
}

\author{
Child adjustment to separation or divorce of parents
}

\author{
Hélder Silva Raposoํㅜ, Barbara Fernandes de Carvalho Figueiredo², Diogo Jorge Pereira do Vale Lamela², \\ Rui Alexandre Nunes-Costa ${ }^{1}$, Maria Conceição Castro ${ }^{1}$, Joana Prego ${ }^{1}$
}

${ }^{1}$ Mestrando em Psicologia Clínica da Universidade do Minho (UM), Portugal.

2 Doutora pela UM, professora-associada do Departamento de Psicologia da UM, coordenadora da Unidade dos Estudos do Divórcio e Intervenção da UM.

3 Doutorando no Programa Doutoral em Psicologia Clínica da UM, professor-assistente na Escola Superior de Educação de Viana do Castelo, Portugal.

Recebido: 26/11/2009 - Aceito: 27/1/2010

\begin{abstract}
Resumo
Objetivo: O presente artigo procura relacionar e analisar evidências empíricas e teóricas sobre o impacto e os fatores associados ao impacto da separação ou divórcio dos pais no ajustamento da criança. Método: Realizou-se uma revisão agregativa da literatura, recorrendo às palavras-chave "divorce adjustment", "child divorce" e "divorce impact", nas bases de dados JSTOR, PsycInfo, SciELO e Medline e em livros da especialidade. Resultados: Respostas adaptativas e desadaptativas da criança são descritas, assim como potenciais resultados positivos. São principalmente discutidos os fatores mediadores e moderadores frequentemente referenciados na literatura no impacto da separação ou divórcio dos pais no ajustamento da criança. Entre esses estão: características da criança, problemas financeiros, sintomatologia psicopatológica dos pais, qualidade das práticas parentais e conflito interparental. Conclusão: Com base nas evidências empíricas e perspectivando o divórcio como uma transição desenvolvimental, levantamos a hipótese de que os problemas de ajustamento apresentados pelas crianças com pais divorciados possam ser mais bem explicados por outros fatores do que pelo divórcio/separação per se. Finalmente assumimos uma inovação conceptual de que essa transição familiar pode significar uma oportunidade de crescimento e de promoção desenvolvimental.
\end{abstract}

Raposo HS, et al. / Rev Psiq Clín. 2011;38(1):29-33

Palavras-chave: Ajustamento, criança, divórcio, coparentalidade, conflito interparental.

\begin{abstract}
Objective: Current theoretical and empirical evidences about the impact and the factors associated with the impact of parents' separation/divorce in children's adjustment were related and examined. Method: An aggregative literature review was made, using the keywords "divorce adjustment", "child divorce" e "divorce impact", from the databases JSTOR, PsycInfo, SciELO, and Medline and from reference books. Results: Child unadaptive and adaptive responses are described, as well as potential positive results. Mediators and moderators factors of the impact of parents' separation/divorce in children's adjustment often referenced in literature are mainly discussed. Among these are: characteristics of children, financial problems, parents' psychopathological symptoms, quality of parenting practices and inter-parental conflict. Discussion: Based on the existing results, and considering the divorce as a developmental transition, we put a hypothesis that adjustment problems showed by children's of divorce can be better explained by other factors than the divorce/separation by itself. Finally we assume a conceptual innovation that this familiar transition can represent a growth opportunity and developmental promotion.
\end{abstract}

Raposo HS, et al. / Rev Psiq Clín. 2011;38(1):29-33

Keywords: Adjustment, children, divorce, co-parenting, inter-parental conflict.

\section{Introdução}

O divórcio tem sido descrito como um evento estressante para as famílias, nomeadamente porque implica uma série de mudanças e ajustamentos na vida dos pais e das crianças ${ }^{1-4}$. Como outras situações familiares estressantes, o divórcio aumenta a probabilidade de pais e crianças evidenciarem mal-estar psicológico ${ }^{1,2,5-7}$. Reações adversas em vários níveis, assim como sintomas que são comuns nessa fase de transição, têm sido descritas nos mais variados estudos ${ }^{6-11}$.

A literatura aponta que crianças de famílias divorciadas estão em risco de apresentar problemas de ajustamento ${ }^{6,12,13}$. A metanálise de Amato e Keith ${ }^{5}$ mostra que crianças de pais divorciados, quando comparadas com crianças que vivem em famílias intactas, exibem indicadores de menor bem-estar, incluindo pior realização acadêmica, ajustamento psicológico, autoconceito, relações sociais e qualidade de relacionamento com o pai e a mãe. Posteriormente, o autor realizou novas análises englobando os resultados dos estudos publicados nos anos $1990^{6}$ e comparando-os com os resultados da metanálise anterior, mostrando que os filhos de pais divorciados, em comparação com os filhos de pais casados, continuam a exibir piores resultados: no ajustamento psicológico, na realização acadêmica, quanto ao nível comportamental, do autoconceito e relações sociais. O autor refere ainda que as diferenças entre os dois grupos foram mais acentuadas nos anos 1990, em comparação aos anos 19806. Mais recentemente, Amato ${ }^{14}$ sinaliza, ainda, que as crianças que crescem com pais casados têm menos probabilidade de experienciar uma grande variedade de problemas cognitivos, emocionais e sociais, não somente durante a infância, mas também na idade adulta. Questionando-se sobre a origem dessas diferenças, o autor sugere que as crianças que crescem em famílias estáveis, com ambos os pais, apresentam níveis mais elevados de qualidade de vida, beneficiam-se de maiores níveis de efetividade parental, coparentalidade, proximidade emocional com ambos os pais e estão sujeitas a menos acontecimentos e situações estressantes.

A presente revisão da literatura procurou enunciar e agregar dados relativos aos fatores mediadores/moderadores frequentemente reportados na literatura, associando-os ao impacto que o divórcio ou separação dos pais pode ter no ajustamento da criança ao divórcio, nomeadamente no desenvolvimento de respostas adaptativas e desadaptativas. 


\section{Método}

Na presente revisão agregativa da literatura, recorreu-se às bases de dados JSTOR, PsycInfo, SciELO e Medline, utilizando os unitermos "divorce adjustment", "child divorce" e "divorce impact". Foram selecionados os artigos mais representativos sobre o tema e dada especial atenção aos contributos de investigadores de referência internacional. Também foram consultados alguns livros de referência que se justificassem no enquadramento do presente artigo de revisão.

\section{Resultados e discussão}

\section{Mediadores/moderadores dos problemas de ajustamento da criança}

No modelo biopsicossocial do impacto da dissolução conjugal proposto por Troxel e Matthews ${ }^{15}$, em 2004, a separação é um estressor familiar que tem, por um lado, um efeito desorganizador das práticas parentais e origina, por outro lado, a redução da segurança econômica da família. Essas dimensões, associadas a fatores de vulnerabilidade biológica, familiar, interpessoal e social, contribuem para a desregulação e insegurança emocionais da criança. Reações comuns podem traduzir-se em problemas de saúde física e psicológica na presença dessas vulnerabilidades. Segundo Nunes-Costa et al. ${ }^{16}$, a separação ou divórcio dos pais pode desencadear a diminuição da saúde física ${ }^{17}$ e psicológica, mas não significa por si só a desadaptação desenvolvimental da criança. Só quando se associa a outros fatores de risco, como o conflito interparental, desencadeia trajetórias desenvolvimentais caracterizadas por uma inadequada adaptação, com piores níveis de saúde física, sintomatologia psicopatológica, pior rendimento acadêmico e comportamentos de risco. Também Lansford ${ }^{10}$ alerta de que nem todas as crianças vivenciam trajetórias de desadaptação, sendo a resposta determinada por circunstâncias individuais. A título de exemplo, Kelly e Emery ${ }^{18}$ referem como fatores protetores no ajustamento da criança ao divórcio: a competência e a adequação dos pais na disputa pela custódia e a qualidade das práticas parentais, a parentalidade apropriada do pai sem a custódia e a diminuição dos conflitos parentais durante o divórcio. Estes últimos, quando presentes, podem amenizar e amortecer o impacto do divórcio ou separação, e com maior probabilidade existe uma adequada adaptação desenvolvimental da criança.

A questão de se o divórcio ou separação parental per se e as condições que com frequência lhe estão associadas determinam os piores resultados das crianças, evidenciados na literatura, é por conseguinte relevante. A ideia de que o divórcio e a separação dos pais per se determinam quase "invariavelmente" piores resultados psicológicos na criança é cada vez menos enfatizada na literatura. $\mathrm{O}$ interesse atual dos investigadores dirige-se para a procura dos fatores, modelos e processos que melhor permitam explicar o modo como o divórcio pode afetar o ajustamento da criança, em vez da comparação do ajustamento entre crianças de famílias divorciadas e intactas. A investigação procura, assim, conciliar duas posições extremistas: 1) de que o divórcio é debilitante e provoca efeitos negativos e duradouros no ajustamento a longo prazo da criança (Wallerstein et al. ${ }^{19}$ ); e 2) de que não existem efeitos negativos a longo prazo no ajustamento da criança (Harris ${ }^{20}$ ). Tendencialmente, chega-se à conclusão de que o divórcio pode acarretar efeitos negativos no ajustamento da criança, na maior parte das vezes transitórios, cujas magnitude e extensão no tempo dependem de outras circunstâncias, tal como se discute a seguir.

Por exemplo, Amato e Keith 5 , tomando em consideração os processos familiares e outras variáveis mediadoras, realçam a presença de cinco fatores associados ao pior ajustamento da criança à separação ou divórcio dos pais: 1) dificuldades da criança (como o temperamento e a idade); 2) diminuição da segurança financeira no período que segue a dissolução conjugal; 3) quadros psicopatológicos nos pais, com especial relevância para a depressão; 4) coparentalidade conflituosa; e 5) intensidade, tonalidade e frequência do conflito interparental antes e após a separação.

\section{Características da criança}

Temperamento e nível de desenvolvimento são dimensões intrínsecas à criança, essenciais na compreensão do processo de adaptação à separação ou divórcio parental ${ }^{21-25}$. Crianças com temperamento fácil, inteligentes, responsáveis e socialmente sensíveis evidenciam mais competência de adaptação positiva a essa transição familiar ${ }^{24}$. Dimensões como a autoestima, a competência cognitiva e a autonomia da criança, associadas ao sistema de suporte social, também estão positivamente ligadas a uma maior adaptação da criança18.

Quando se procura compreender o impacto que o divórcio pode ter na criança, é ainda essencial considerar as diferenças desenvolvimentais decorrentes da idade. O divórcio não implica forçosamente maior impacto numa dada idade, mas sim efeitos diferentes consoante o estágio desenvolvimental da criança. No entanto, quando se isola o nível desenvolvimental de outras variáveis moderadoras, observa-se que quanto mais elevado e integrado o nível de desenvolvimento da criança, melhores os índices de adaptação à separação dos pais ${ }^{6}$.

Alguns estudos concluíram que crianças em idade pré-escolar apresentam maior risco ecológico e desenvolvimental para trajetórias sociais e emocionais desadaptadas, em comparação com crianças de mais idade ${ }^{18}$. Algumas tarefas desenvolvimentais podem ficar comprometidas pela incapacidade da criança, relacionada com a idade, de compreender as mudanças e o significado do conflito e do divórcio parental ${ }^{26}$. A imaturidade das suas estruturas cognitivo-emocionais faz com que sejam menos capazes de avaliar realisticamente as causas, os processos e as consequências da separação e centralizem em si a responsabilidade pela ruptura entre os pais, ao que se alia uma menor competência para conseguir o suporte necessário à diminuição do seu nível de aflição.

\section{Problemas financeiros}

Um dos principais fatores de risco, relacionado com o pior ajustamento da criança à separação ou divórcio dos pais, é a diminuição da segurança financeira. As dificuldades econômicas que surgem após a dissolução marital 27,28 parecem ter impacto significativo na redução dos níveis de bem-estar e sucesso acadêmico da criança ${ }^{29,30}$. As mudanças financeiras têm impacto direto, mas também indireto, sobre a criança.

Um impacto direto porque menos recursos econômicos podem significar para a criança menor qualidade de vida (p. ex.: viver em bairros com problemas), mudar de escola e ter menos oportunidades educacionais, interferindo na sua realização acadêmica ${ }^{30,31}$. Tendencialmente, os pais que ficam com a guarda sentem um acentuado declínio nos recursos econômicos disponíveis para responder às necessidades da família. A redução do rendimento familiar tem impacto no bem-estar real e subjetivo da criança, traduzido no decréscimo de recursos financeiros disponíveis para a saúde, educação, atividades extracurriculares, acesso a bens culturais e de entretenimento e aquisição de produtos utilizados diariamente ${ }^{5,28,32}$. Os pais mais desprotegidos do ponto de vista econômico podem estar impossibilitados de proporcionar as condições de vida necessárias aos filhos. Caso as dificuldades financeiras sejam crônicas, podem, ainda, não permitir o acesso a atividades essenciais ao desenvolvimento cognitivo e social da criança. Já quanto ao impacto indireto, as dificuldades econômicas afetam muitas vezes o ajustamento pós-divórcio da mãe, influenciando o seu estado de humor, funcionamento e capacidade para providenciar cuidados apropriados à criançá2,33. Outros estudos defendem que as dificuldades financeiras amplificam as consequências negativas da separação ou divórcio dos pais. Hetherington et al. ${ }^{21}$, por exemplo, referem que a dificuldade econômica em acréscimo ao estresse do divórcio é a maior causa para as dificuldades da criança.

\section{Sintomatologia psicopatológica dos pais}

As alterações na estrutura familiar expõem a criança a um contexto de interação em que predominam estressores psicossociais, que po- 
dem dificultar a sua competência para se ajustar ao divórcio dos pais. O modo como os pais lidam com o divórcio, principalmente os seus níveis de sintomatologia psicopatológica, tem sido relacionado com os níveis de ajustamento da criança ${ }^{10,34}$.

A epidemiologia dos transtornos mentais, que vem se ocupando de forma crescente, nos últimos 25 anos, dos chamados eventos de vida produtores de estresse, considera o divórcio um rito de passagem em que as mudanças implicadas são produtoras de estresse ${ }^{35}$. Esse estresse, por sua vez, aumenta a probabilidade de desadaptação psicossocial parental e vulnerabilidade psicopatológica ${ }^{16}$.

Du Rocher Schudlich e Cummings ${ }^{34}$ indicam que o bem-estar parental está associado ao ajustamento ao divórcio, por meio de processos familiares mediadores específicos e distintos, como o conflito parental e a parentalidade. Além disso, a segurança emocional da criança, no contexto de diferentes estilos de conflito marital, é mediada pela relação entre o mal-estar parental e os problemas de ajustamento da criança. Se o conflito marital e o divórcio aumentam os níveis de ansiedade, depressão e estresse parental, diminuem consequentemente a efetividade dos pais e podem, por sua vez, afetar negativamente o ajustamento da criança. Por exemplo, a existência de depressão parental aumenta a probabilidade de diminuição da qualidade de prestação de cuidados instrumentais e emocionais ${ }^{32}$. As mães com sintomatologia depressiva exibem mais afeto negativo, mais comportamentos negligentes, mais comportamentos hostis, menor consistência educativa, menos comportamentos parentais positivos, menores cuidados com a saúde da criança, menor disponibilidade emocional e mais comportamentos parentais de risco ${ }^{36}$. Como resultado, as crianças de pais separados deprimidos ou ansiosos apresentam maior probabilidade de desenvolver perturbações de depressão e ansiedade, mais comportamentos oposicionais, menor autoestima, pior comportamento social, pior rendimento acadêmico, maiores défices de atenção e maiores dificuldades de relacionamento interpessoal ${ }^{37}$.

Também Lansford ${ }^{10}$ considera o rendimento econômico, o conflito interparental, as práticas parentais e o bem-estar dos pais como mediadores da relação divórcio e ajustamento da criança. Esses quatro fatores podem amortecer a associação existente entre divórcio parental e dificuldades de ajustamento da criança. Um processo de divórcio harmonioso entre os pais, tendo em vista o interesse superior da criança, reduz as dificuldades de ajustamento dos filhos. O bem-estar dos pais é aqui referido como um possível mediador no ajustamento da criança ao divórcio. Dessa forma, compreende-se que os fatores protetores do ajustamento da criança ao divórcio e redutores do risco estejam relacionados com dimensões do ajustamento parental.

\section{Práticas parentais}

Alguns estudos têm demonstrado que as práticas parentais são particularmente críticas e suscetíveis de explicar os efeitos da separação ou divórcio dos pais na criança ${ }^{38}$. Para Amato $^{14}$, a qualidade da parentalidade é um dos melhores preditores do bem-estar social e emocional da criança. No período inicial da dissolução conjugal, os pais estão mais centrados no seu ajustamento às mudanças familiares ${ }^{39}$, por isso tanto a consistência e a concordância nos cuidados quanto a partilha de um estilo parental democrático podem estar dificultadas. Um estilo parental democrático partilhado por ambos elementos da díade (por exemplo, quando as mães facilitam e incentivam a guarda partilhada com o ex-cônjuge) facilita o bom ajustamento da criança ${ }^{40}$. Caso contrário, quando os pais divergem no estilo parental, confrontando a criança com mensagens educativas inconsistentes, $o$ estresse e os problemas de internalização aumentam.

A coparentalidade cooperativa, definida pelo envolvimento conjunto e recíproco de ambos os pais na educação, cuidados e decisões sobre a vida dos filhos ${ }^{41}$, é igualmente decisiva no ajustamento da criança ao divórcio. Os pais que exercem uma relação de coparentalidade cooperativa, ao imprimirem prioridade ao bem-estar dos filhos, mantêm uma relação construtiva, com novas fronteiras, novos papéis parentais flexíveis e maleáveis entre si, com vista a resposta às necessidades da criança. Nesse sentido, os pais partilham a responsabilidade pela educação dos filhos e cooperam na tomada de decisão.

Existem três variações da coparentalidade ${ }^{42}$, sendo a coparentalidade cooperativa, em vez da coparentalidade conflituosa ou descomprometida, a que melhor propicia o ajustamento da criança. Maior cooperação, respeito e comunicação na díade parental quanto às necessidades dos filhos, maior envolvimento do pai sem a guarda parental (caracterizado por uma forte ligação emocional consistente) e estilo parental democrático influenciam e promovem o bem-estar da criança, em dimensões como o rendimento escolar e o estado de saúde ${ }^{43}$.

Contudo, na sequência da separação ou divórcio parental, o risco de relações coparentais descomprometidas é elevado ${ }^{4,42,43}$. As relações descomprometidas caracterizam-se por distância física entre os pais, dificuldades em isolar a relação conjugal da atual relação coparental e diminuição do envolvimento na vida da criança do pai não detentor da guarda ${ }^{44}$. Por sua vez, quando os pais apresentam um padrão coparental disruptivo, as relações são paralelas e pouco consistentes, existem altos índices de conflituosidade que, consequentemente, minam a percepção da criança sobre a aliança parental.

\section{Conflito interparental}

O conflito interparental é considerado o fator de risco com maior impacto no ajustamento da criança à separação ou divórcio dos pais ${ }^{45,10}$. O conflito interparental - manifestado pela raiva, hostilidade, desconfiança, linguagem agressiva, agressão física, dificuldades de cooperação nos cuidados e comunicação com os filhos etc. ${ }^{44}$ - cria um ambiente familiar estressante, suscitando reações de estresse, tristeza e insegurança na criança ${ }^{46}$. Esse ambiente não é propício ao desenvolvimento adequado da criança e tem um impacto negativo no seu ajustamento psicológico ${ }^{47}$.

A desarmonia conjugal é também um fator de risco psicossocial encontrado com maior frequência entre as famílias com pais alcoólicos, existindo uma elevada taxa de divórcios e separações ${ }^{48}$. Esse fato aponta para que as crianças expostas ao divórcio/separação dos pais, em que predominam os conflitos interparentais, estão mais suscetíveis de viver em ambientes familiares desestruturantes e estressantes coexistentes com alcoolismo parental.

Relações parentais conflituosas, quando acontece o emaranhamento dos problemas conjugais na relação parental, têm não só efeitos diretos no funcionamento psicológico da criança, mas também efeitos indiretos, dado que interfere na qualidade do comportamento parental. O conflito interparental coloca os pais numa posição menos efetiva para lidar com os filhos ${ }^{10}$. Além do que, quando as crianças são envolvidas nos conflitos parentais, acontece a deterioração das relações pais-filhos ${ }^{49}$. Práticas e rotinas contaminadas pelo elevado conflito entre os pais traduzem-se, na maioria das vezes, em disciplina permissiva e inconsistente, volatilidade emocional, elevados índices de hostilidade e impulsividade educativa e menor responsabilidade e disponibilidade emocional.

Compreende-se desse modo 1) porque as crianças de famílias intactas, em que existem elevados níveis de conflitos, tendem a mostrar problemas similares aos das crianças de pais divorciados e 2) porque os conflitos parentais pós-divórcio estão associados a baixos níveis de bem-estar nas crianças ${ }^{5,6}$. Com efeito, as crianças cujos pais estão envolvidos durante longos períodos de tempo em disputas judiciais sobre a regulação do poder parental apresentam pior ajustamento ao divórcio ${ }^{50,51}$. No estudo de Bing et al. ${ }^{51}$, as famílias que experienciam níveis mais elevados de conflito, medidos pelos níveis de envolvimento judicial, mostram menor resolução positiva do divórcio e as crianças, menos competências de coping.

A investigação ilustra que a percepção da criança acerca do grau de conflito interparental funciona como um mediador na presença/ ausência de sintomas psicopatológicos: quanto maior a percepção da destrutibilidade dos conflitos entre os pais, maior o risco para problemas de ajustamento na criança ${ }^{12}$. Crianças envolvidas em 
separações altamente conflituosas apresentam mais problemas de externalização, quando comparadas com crianças que experienciam separações parentais com baixos níveis de conflito ${ }^{45}$. Segundo Emery ${ }^{52}$, processos familiares, como a parentalidade inadequada, conflito interparental e desestruturação da estrutura familiar, explicam muitos dos comportamentos de externalização encontrados entre as crianças dos pais divorciados.

\section{Principais conclusões}

O presente artigo pretendeu enunciar e refletir sobre o impacto e os fatores associados ao impacto da separação/divórcio dos pais no ajustamento da criança. Da análise da literatura, surgem algumas conclusões relevantes. A primeira é que o divórcio dos pais se associa a problemas transitórios de ajustamento nas crianças, as quais diminuem os resultados desenvolvimentais ${ }^{23}$ e estão duas vezes mais em risco de desenvolver problemas do que a população não divorciada $^{44}$ nos dois primeiros anos após a dissolução conjugal. A maioria dos estudos revisitados aponta que a magnitude e a duração dos problemas que a criança pode exibir decorrem não diretamente da mudança na estrutura familiar, mas dos conflitos que a acompanha ${ }^{53}$, assim como do modo como os pais se ajustam ao divórcio, nomeadamente da presença de sintomatologia psicopatológica, o que largamente interfere na sua competência parental.

A segunda conclusão relaciona-se com a impossibilidade empírica de associar o divórcio a uma maior probabilidade de a criança apresentar problemas de ajustamento a médio ou longo prazo. Como já sublinhamos, essa associação não parece ser simples ${ }^{16} \mathrm{e}$ tende a surgir por meio da inclusão de variáveis mediadoras e moderadoras na equação, que emprestam poder às conclusões da maioria dos estudos. Aparentemente, a configuração estrutural da família não é responsável direta pela qualidade do ajustamento da criança. Manutenção do bem-estar financeiro da família, ajustamento psicológico dos pais, coparentalidade positiva e resolução de conflitos interparentais são as dimensões primordiais.

Ao longo das últimas décadas, a investigação prevalente é rica em enunciar e descrever o quão disruptivo pode ser o divórcio dos pais para o desenvolvimento da criança, colocando em segundo plano o reverso desse processo, isto é, o potencial de enriquecimento que essa transição familiar pode comportar. Assumir a possibilidade de crescimento não significa a negação do risco acrescido a que crianças de pais divorciados estão expostas. Sustentando-se nos perfis de adaptação e desenvolvimento obtidos por meio dos dados recolhidos em estudos longitudinais, que avaliaram comparativamente as trajetórias desenvolvimentais de crianças e adolescentes oriundas de famílias intactas (com e sem conflito), famílias com pais divorciados e famílias reconstituídas, Hetherington e Kelly constataram que as crianças de pais divorciados situavam-se com frequência nos clusters de crianças com piores ou melhores resultados desenvolvimentais ${ }^{23}$. A esses dados somam-se outros estudos que concluíram que a linearidade divórcio igual a problemas de adaptação é falaciosa e não reflete com exatidão os resultados da investigação empírica ${ }^{18,54}$. Baseados nessas conclusões empíricas, levantamos a hipótese de que os problemas de ajustamento apresentados por parte das crianças com pais divorciados possam ser mais bem explicados por outros fatores do que pelo divórcio per se. O presente artigo teve como finalidade apresentar e descrever esses principais fatores com relevância na compreensão dos padrões de ajustamento da criança a essa transição familiar.

Argumentamos ainda que, apesar de esses fatores apresentarem maior saliência nas famílias de pais separados/divorciados, são transversais a qualquer configuração familiar. Por outras palavras, a conjugação sequencial desses fatores prediz os níveis de adaptação da criança, independentemente da estrutura e arranjo familiares ${ }^{55,56}$. Com este artigo pretendeu-se sublinhar que o divórcio não se apresenta tão nefasto para a maioria das crianças que o experienciam. Por isso, é necessário, como agenda futura, iniciar uma mudança epistemológica e centrar parte dos recursos científicos nos produtos positivos do divórcio. É hoje visível o surgimento de estudos e posições teóricas que assentam nessa proposição conceptual. Pers- pectivando o divórcio como uma transição desenvolvimental, tal transição deve ser lida e analisada à luz dos processos de mudança desenvolvimentais. Isso obriga à utilização dos mapas conceptuais e metodológicos da ciência desenvolvimental. Dessa forma, o estudo do impacto do divórcio, em primeiro lugar, deve assumir este estressor como um processo sistêmico e dinâmico, sublinhando as funções da plasticidade no estudo do desenvolvimento humano, e, em segundo lugar, definir a adaptação como resultado da interação de múltiplos mediadores psicossociais, condicionada por características individuais e pelo contexto desenvolvimental, o que invalida certezas $a$ priori das trajetórias desenvolvimentais pós-divórcio.

São cada vez mais visíveis as inovações conceptuais e empíricas que alicerçam a possibilidade de identificar e decalcar diferentes trajetos desenvolvimentais após a dissolução conjugal dos pais, e esse evento não é, por um lado, obrigatoriamente sinônimo de desajustamento crônico e, por outro lado, poderá ser um momento de promoção desenvolvimental. Dessa forma, a resiliência deve igualmente ser pensada, uma vez que, efetivamente, a grande maioria das crianças que vivenciam o divórcio dos pais apresenta um bom ajustamento e não demonstra vários ou permanentes problemas emocionais ou comportamentais ${ }^{6,18}$.

\section{Referências}

1. Amato PR. Children's adjustment to divorce: theories, hypotheses, and empirical support. J Marriage Fam. 1993;55:23-38.

2. Amato PR. The consequences of divorce for adults and children. J Marriage Fam. 2000;62:1269-87.

3. Brown SL. Family structure transitions and adolescent well-being. Demography. 2006;43:447-61.

4. Hetherington EM. An overview of the Virginia longitudinal study of divorce and remarriage with a focus on early adolescence. J Fam Psychol. 1993;7:39-56.

5. Amato PR, Keith B. Parental divorce and the well-being of children: a meta-analysis. Psychol Bull. 1991;110:26-46.

6. Amato PR. Children of divorce in the 1990s: an update of the Amato and Keith (1991) meta-analysis. J Fam Psychol. 2001;15:355-70.

7. Sarrazin J, Cyr F. Parental conflicts and their damaging effects on children. J Divorce \& Remarriage. 2007;47:77-93.

8. Haslee SLA. The impact of divorce on children: implications for counseling. Masalah Pendidikan. 2007;30:161-8.

9. Huurre T, Junkkari H, Aro H. Long-term psychosocial effects of parental divorce: a follow-up study from adolescence to adulthood. Eur Arch Psychiatry Clin Neurosci. 2006;256:256-63.

10. Lansford JE. Parental divorce and children's adjustment. Perspect Psychol Sci. 2009;4:140-52.

11. Sirvanli-Ozen D. Impacts of divorce on the behavior and adjustment problems, parenting styles, and attachment styles of children. J Divorce \& Remarriage. 2005;43:127-51.

12. Schick A. Behavioral and emotional differences between children of divorce and children from intact families: clinical significance and mediating processes. Swiss J Psychol. 2002;61:5-14.

13. Lansford JE, Malone PS, Castellino DR, Dodge KA, Pettit, GS, Bates JE. Trajectories of internalizing, externalizing, and grades for children who have and have not experienced their parents' divorce. J Fam Psychol. 2006;20:292-301.

14. Amato PR. The impact of family formation change on the cognitive, social, and emotional well-being of the next generation. Future Child. 2005;15:75-96.

15. Troxel WM, Matthews KA. What are the costs of marital conflict and dissolution to children's physical health? Clin Child Fam Psychol Rev. 2004;7:39-57.

16. Nunes-Costa RA, Lamela JPV, Figueiredo BFC. Adaptação psicossocial e saúde física em crianças de pais separados. Rev Pediatr. 2009;85:385-96.

17. Marques AH, Solis ACO, Lotufo Neto F, Lotufo RFM, Prado EBA. Estresse, depressão, alterações imunológicas e doença periodontal. Rev Psiquiatr Clín. 2001;28(5):266-73.

18. Kelly J, Emery R. Children's adjustment following divorce: risk and resilience perspectives. Fam Relat. 2003;52:352-62.

19. Wallerstein JS, Lewis JM, Blakeslee S. The unexpected legacy of divorce: a 25 year landmark study. New York: Hyperion; 2002. 
20. Harris JR. The nurture assumption: why children turn out the way they do. New York: Free Press; 1998.

21. Hetherington EM, Bridges MI, Glendessa M. What matters? What does not? Five perspectives on the association between marital transitions and children's adjustment. Am Psychol. 1998;53:167-84.

22. Hetherington EM, Stanley-Hagan M, Anderson ER. Marital transitions: a child's perspective. Am Psychol. 1989;44:303-12.

23. Hetherington EM, Kelly J. For better or for worse: divorce reconsidered. New York: Norton; 2002.

24. Hetherington EM. Divorce and the adjustment of children. Pediatr Rev. 2005;26:163-9.

25. Lengua LJ, Kovacs EA. Bidirectional associations between temperament and parenting and the prediction of adjustment problems in middle childhood. Appl Dev Psychol. 2005;26:21-38.

26. Burke S, McIntosh J, Gridley H, editors. Parenting after separation: a literature review prepared for the Australian psychological society [Internet]. 2007; [cited 2009 May 22]. Available from: http://www.psychology.org. au/publications/statements/parenting_separation/.

27. McManus PA, DiPrete TA. Losers and winners: the financial consequences of separation and divorce for men. Am Soc Rev. 2001;66:246-68.

28. Teachman J, Paach K. Financial impact of divorce on children and their families. Future Child. 1994;4:63-83.

29. Lerman RI. Marriage and the economic well-being of families with children: a review of the literature [Internet]. Washington, DC: Urban Institute Press; 2002; [cited 2009 May 31]. Available from: http://www. urban.org/publications/410541.html.

30. Fischer T. Parental divorce and children's socio-economic success: conditional effects of parental resources prior to divorce, and gender of child. Sociology. 2007;41:475-95.

31. Couch KA, Lillard DR. Divorce, educational attainment and the earning mobility of sons. J Fam Econ Issues. 1997;18:231-45.

32. Carlson M, Corcoran M. Family structure and children's behavioral and cognitive outcomes. J Marriage Fam. 2001;63:779-92.

33. Buchanan CM, Maccoby EE, Dornbusch SM. Caught between parents: adolescent's experience in divorced homes. Child Dev. 1991;62:1008-29.

34. Du Rocher Schudlich TD, Cummings EM. Parental dysphoria, marital conflict, and parenting: relations with children's emotional security and adjustment. J Abnorm Child Psychol. 2007;35:627-39.

35. Coutinho ESF, Almeida-Filho N, Mari JJ. Fatores de risco para morbidade psiquiátrica menor: resultados de um estudo transversal em três áreas urbanas do Brasil. Rev Psiquiatr Clín. 1999;26(5).

36. Lovejoy MC, Graczyk PA, O’Hare E, Neuman G. Maternal depression and parenting behavior: a meta-analytic review. Clin Psychol Rev. 2000;20:561-92.

37. Pilowsky D, Wickramaratne P, Nomura Y, Weissman M. Family discord, parental depression, and psychopathology in offspring: 20-year follow up. J Am Acad Child Adolesc Psychiatry. 2006;45:425-60.

38. Wolchik SA, Wilcox KL, Tein JY, Sandler IN. Maternal acceptance and consistency of discipline as buffers of divorce stressors on children's. J Abnorm Child Psychol. 2000;28:87-102.
39. Kelly JB. Children's adjustment in conflicted marriage and divorce: a decade review of research. J Am Acad Child Adolesc Psychiatry. 2000;39:963-73.

40. Campana K, Henderson S, Stolberg A, Schum L. Paired maternal and parental parenting styles, child custody and children's emotional adjustment to divorce. J Divorce \& Remarriage. 2008;48:1-20.

41. Macie KM. Influence of co-parenting and marital status on young adult adjustment [these]. Virginia: Virginia Commonwealth University; 2002.

42. Maccoby E, Depner C, Mnookin R. Coparenting in the second year after divorce. J Marriage Fam. 1990;52:141-55.

43. Hetherington EM. The influence of conflict, marital problem solving and parenting on children's adjustment in nondivorced, divorced and remarried families. In: Clarke-Stewart A, Dunn J, editors. Families count: effects on child and adolescent development. New York: Cambridge University Press; 2006, p. 203-37.

44. McIntosh JE. Enduring conflict in parental separation: pathways of impact on child development. J Fam Studies. 2003;9:63-80.

45. Buchanan $\mathrm{C}$, Heiges $\mathrm{K}$. When conflict continues after the marriage ends: effects of postdivorce conflict on children. In: Grych H, Fincham F, editors. Interparental conflict and child development. New York: Cambridge University Press; 2001, p. 337-62.

46. Maccoby E, Martin JA. Socialization in the context of the family: parent-child interaction. In: Hetherington EM, editor. Handbook of child psychology: socialization, personality, and social development. New York: Wiley; 1983, p. 1-101.

47. Emery RE. Interparental conflict and the children of discord and divorce. Psychol Bull. 1982;92:310-30.

48. Furtado EF, Lauch M, Scmidt M. Estudo Longitudinal prospectivo sobre risco de adoecimento psiquiátrico na infância e alcoolismo paterno. Rev Psiquiatr Clín. 2002;29(2):71-80.

49. Amato PR. Marital conflict, the parent-child relationship, and child self-esteem. Fam Relat. 1986;35:103-10.

50. Johnston J. Developing and testing a group intervention for families at impasse. California: Center for the Family in Transition; 1998.

51. Bing NM, Nelson III WM, Wesolowski KL. Comparing the effects of amount of conflict on children's adjustment following parental divorce. J Divorce \& Remarriage. 2009;50:159-71.

52. Emery RE. Marriage, divorce, and children's adjustment. 2. ed. Thousand Oaks, CA: Sage; 1999.

53. Vandewater EA, Lansford JE. Influences of family structure and parental conflict on children's well-being. Fam Relat. 1998;47:323-30.

54. Clarke-Stewart KA, Vandell DL, McCartney K, Owen MT, Booth CL. Effects of parental separation and divorce on very young children. J Fam Psychol. 2000;14:304-26.

55. Hetherington EM, Bridges M, Insabella GM. What matters? What does not? Five perspectives on the association between marital transitions and children's adjustment. Am Psychol. 1998;53:167-84.

56. Clarke-Stewart A, Brentano C. Divorce, causes and consequences. New Haven: Yale University Press; 2006. 